Zoltán Boros*, Institute of Mathematics and Informatics, University of Debrecen, H-4010 Debrecen, Pf. 12, Hungary. e-mail: boros@math.klte.hu

\title{
STRONGLY $\mathbb{Q}$-DIFFERENTIABLE FUNCTIONS
}

\begin{abstract}
A real function is called strongly $\mathbb{Q}$-differentiable if, for every real number $h$, the limit of the ratio $(f(x+r h)-f(x)) / r$ exists whenever $x$ tends to any fixed real number and $r$ tends to zero through the positive rationals. After examining the dependence of strong $\mathbb{Q}$-derivatives on their parameters, we prove that every strongly $\mathbb{Q}$-differentiable function can be represented as the sum of an additive mapping and a continuously differentiable function.
\end{abstract}

\section{Introduction}

It is well known that the graph of a non-linear additive function $\phi: \mathbb{R} \rightarrow \mathbb{R}$ is dense in $\mathbb{R}^{2}$, thus such a function $\phi$ serves as a counterexample for the usual regularity concepts in mathematical analysis. However, one can observe that the difference $\Delta_{h} \phi(x)=\phi(x+h)-\phi(x)$ does not depend on $x$. Therefore, supposing that the real function $g$ has some nice regularity property and $f=$ $g+\phi$, we obtain that the difference function $\Delta_{h} f$ fulfils the same regularity assumption for each value of $h$. The converse of this implication has been proved by de Bruijn [2] for a number of standard regularity properties including continuity and differentiability.

General solutions of the polynomial (and monomial) functional equations have been characterized as functions having vanishing higher order (modified) Dinghas interval derivatives [6], [3]. Non-linear additive functions, as well as

\footnotetext{
Key Words: differentiability, real function, additive function

Mathematical Reviews subject classification: 26A24, 26A30, 39B22

Received by the editors February 7, 2000

* This research has been supported in part by the Hungarian Scientific Research Fund (OTKA) Grant T-030082 and by the Kereskedelmi és Hitelbank Rt. Universitas Foundation.
} 
other discontinuous solutions of those functional equations, serve as examples for that regularity concept.

Nikodem and Páles [5] used an inequality and a weak continuity property in order to characterize the composition of an upper (or lower) semicontinuous strictly monotonic function with an additive mapping. The definition of that continuity property involves a limit of a function as a rational coefficient in its argument tends to 0 from above.

Motivated by these results we will introduce a differentiability property involving positive rational numbers in the denominator of the appropriate difference quotient. We shall call it "strong", because we need a double limit in order to obtain a decomposition theorem which is similar to the ones mentioned above. The set of the positive rationals will be denoted by $\mathbb{Q}^{+}$.

Definition 1. Let $f: \mathbb{R} \rightarrow \mathbb{R}, h, x_{0} \in \mathbb{R}$. If the finite limit

$$
D_{h}^{\mathbb{Q}} f\left(x_{0}\right)=\lim _{\mathbb{R} \times \mathbb{Q}^{+} \ni(x, r) \rightarrow\left(x_{0}, 0\right)} \frac{f(x+r h)-f(x)}{r}=\lim _{\substack{x \rightarrow x_{0} \\ \mathbb{Q}^{+} \ni r \rightarrow 0}} \frac{f(x+r h)-f(x)}{r}
$$

exists, it is called the strong $\mathbb{Q}$-derivative of $f$ at $x_{0}$ in the direction $h$ (where we consider the first limit as the exact definition and the second limit as an abbreviation for the first one, understanding that $x_{0}$ is an admissible value for $x$ ). We shall say that $f$ is strongly $\mathbb{Q}$-differentiable if $D_{h}^{\mathbb{Q}} f\left(x_{0}\right) \in \mathbb{R}$ exists for every $h, x_{0} \in \mathbb{R}$.

This definition is motivated by the following fundamental examples.

Example 1.1. Let $f: \mathbb{R} \rightarrow \mathbb{R}$ be continuously differentiable. Applying Lagrange's middle value theorem it is easy to check that $f$ is strongly $\mathbb{Q}$-differentiable and

$$
D_{h}^{\mathbb{Q}} f\left(x_{0}\right)=f^{\prime}\left(x_{0}\right) h \text { for every } h, x_{0} \in \mathbb{R} .
$$

Let us recall that a mapping $\phi: \mathbb{R} \rightarrow \mathbb{R}$ is additive if

$$
\phi(x+y)=\phi(x)+\phi(y) \text { for every } x, y \in \mathbb{R} .
$$

We shall also apply the well known basic result that every additive function is linear over $\mathbb{Q}[4$, Theorem 5.2 .1 (p. 121)].

Example 1.2. If $f: \mathbb{R} \rightarrow \mathbb{R}$ is additive, then

$$
\frac{f(x+r h)-f(x)}{r}=\frac{f(r h)}{r}=f(h)
$$


for every $h, x \in \mathbb{R}, r \in \mathbb{Q}^{+}$. Hence $f$ is strongly $\mathbb{Q}$-differentiable and

$$
D_{h}^{\mathbb{Q}} f\left(x_{0}\right)=f(h) \text { for every } h, x_{0} \in \mathbb{R} .
$$

It is an immediate consequence of the definition that the set of strongly $\mathbb{Q}$ differentiable functions is a linear space (over $\mathbb{R}$ ) and the mapping $f \mapsto D_{h}^{\mathbb{Q}} f(x)$ is a linear functional for every $h, x \in \mathbb{R}$. However, the following example shows that the square of a strongly $\mathbb{Q}$-differentiable function may fail to inherit this property.

Example 1.3. Let $\phi: \mathbb{R} \rightarrow \mathbb{R}$ be a discontinuous additive function and for $x \in \mathbb{R}$ let $f(x)=(\phi(x))^{2}$. Then

$$
\frac{f(x+r h)-f(x)}{r}=\frac{(\phi(x)+r \phi(h))^{2}-(\phi(x))^{2}}{r}=r(\phi(h))^{2}+2 \phi(x) \phi(h)
$$

for every $h, x \in \mathbb{R}, r \in \mathbb{Q}^{+}$. Keeping $h$ fixed, the first term tends to 0 as $r$ tends to 0 , while the second term has no limit as $x$ tends to any fixed real number unless $\phi(h)=0$. Therefore $f$ is not strongly $\mathbb{Q}$-differentiable.

These examples show that the sum of a continuously differentiable function and an additive mapping is a typical strongly $\mathbb{Q}$-differentiable function. Our main result (Theorem 3.1) states that the converse is also true; namely, that every strongly $\mathbb{Q}$-differentiable function can be decomposed into such a sum.

\section{Basic properties}

In this section, we encounter some interesting properties of strongly $\mathbb{Q}$-differentiable functions and strong $\mathbb{Q}$-derivatives. These properties also motivate and anticipate our main result.

The real open interval with lower limit $a$ and upper limit $b$ will be denoted by $] a, b[$, and we shall write $U(x, \delta)=] x-\delta, x+\delta[$ for the open neighborhood in $\mathbb{R}$ with center $x \in \mathbb{R}$ and radius $\delta>0$.

Proposition 2.1. Let $f: \mathbb{R} \rightarrow \mathbb{R}$ be strongly $\mathbb{Q}$-differentiable, $x_{0} \in \mathbb{R}$, and for $h \in \mathbb{R}$ let $\phi(h)=D_{h}^{\mathbb{Q}} f\left(x_{0}\right)$. Then $\phi: \mathbb{R} \rightarrow \mathbb{R}$ is additive. 
ProOF. For every $h_{1}, h_{2} \in \mathbb{R}$ we have

$$
\begin{aligned}
& \phi\left(h_{1}+h_{2}\right)=D_{h_{1}+h_{2}}^{\mathbb{Q}} f\left(x_{0}\right)=\lim _{\substack{x \rightarrow x_{0} \\
\mathbb{Q}^{+} \ni r \rightarrow 0}} \frac{f\left(x+r\left(h_{1}+h_{2}\right)\right)-f(x)}{r} \\
= & \lim _{\substack{x \rightarrow x_{0} \\
\mathbb{Q}^{+} \ni r \rightarrow 0}} \frac{f\left(x+r h_{1}+r h_{2}\right)-f\left(x+r h_{1}\right)+f\left(x+r h_{1}\right)-f(x)}{r} \\
= & \lim _{\substack{x \rightarrow x_{0} \\
\mathbb{Q}^{+} \ni r \rightarrow 0}} \frac{f\left(x+r h_{1}\right)-f(x)}{r}+\lim _{\substack{x \rightarrow x_{0} \\
\mathbb{Q}^{+} \ni r \rightarrow 0}} \frac{f\left(\left(x+r h_{1}\right)+r h_{2}\right)-f\left(x+r h_{1}\right)}{r} \\
= & D_{h_{1}}^{\mathbb{Q}} f\left(x_{0}\right)+D_{h_{2}}^{\mathbb{Q}} f\left(x_{0}\right)=\phi\left(h_{1}\right)+\phi\left(h_{2}\right),
\end{aligned}
$$

because $x+r h_{1}$ tends to $x_{0}$ as $x$ tends to $x_{0}$ and $r$ tends to 0 .

Proposition 2.2. Let $f: \mathbb{R} \rightarrow \mathbb{R}$ be strongly $\mathbb{Q}$-differentiable, $h_{0} \in \mathbb{R}$, and for $x \in \mathbb{R}$ let $\psi(x)=D_{h_{0}}^{\mathbb{Q}} f(x)$. Then $\psi: \mathbb{R} \rightarrow \mathbb{R}$ is continuous.

Proof. Fix an arbitrary $\varepsilon>0$. According to the definition, for every $u \in \mathbb{R}$ there exists $\delta(u)>0$ such that

$$
\left|\frac{f\left(y+r h_{0}\right)-f(y)}{r}-D_{h_{0}}^{\mathbb{Q}} f(u)\right|<\frac{\varepsilon}{2}
$$

for all $y \in \mathbb{R}, r \in \mathbb{Q}^{+}$with $|y-u|<\delta(u)$ and $|r|<\delta(u)$.

If $x_{0} \in \mathbb{R}$ is given, take $x \in \mathbb{R}$ satisfying $\left|x-x_{0}\right|<\delta\left(x_{0}\right) / 2$, and choose $y \in$ $\mathbb{R}, r \in \mathbb{Q}^{+}$such that $|y-x|<\min \left\{\delta(x), \delta\left(x_{0}\right) / 2\right\}$ and $|r|<\min \left\{\delta(x), \delta\left(x_{0}\right)\right\}$. Then we also have $\left|y-x_{0}\right|<\delta\left(x_{0}\right)$. Therefore (2) holds for $u=x$ and also for $u=x_{0}$. This yields

$$
\left|\psi(x)-\psi\left(x_{0}\right)\right|=\left|D_{h_{0}}^{\mathbb{Q}} f(x)-D_{h_{0}}^{\mathbb{Q}} f\left(x_{0}\right)\right|<\varepsilon .
$$

Theorem 2.3. Suppose that $f: \mathbb{R} \rightarrow \mathbb{R}$ is strongly $\mathbb{Q}$-differentiable, $h \in$ $\mathbb{R} \backslash\{0\}, \alpha, \beta \in \mathbb{R}$ with $\alpha<\beta$, and $M \in \mathbb{R}$ such that

$$
\left|D_{h}^{\mathbb{Q}} f(x)\right| \leq M \text { for every } x \in[\alpha, \beta] .
$$

If $u, v \in[\alpha, \beta]$ satisfy $(v-u) / h \in \mathbb{Q}$, then

$$
|f(v)-f(u)| \leq \frac{M}{|h|}|v-u| .
$$


Proof. Let $\varepsilon>0$ be arbitrarily given. Again, by definition, for every $x \in$ $[\alpha, \beta]$ there exists $\delta(x)>0$ such that

$$
\left|\frac{f(y+r h)-f(y)}{r}-D_{h}^{\mathbb{Q}} f(x)\right|<\varepsilon
$$

for all $y \in U(x, \delta(x)), r \in \mathbb{Q} \cap] 0, \delta(x) /|h|[$. Obviously,

$$
[\alpha, \beta] \subset \bigcup_{x \in[\alpha, \beta]} U(x, \delta(x))
$$

and the interval $[\alpha, \beta]$ is compact. Hence there exist $k \in \mathbb{N}$ and $x_{j} \in[\alpha, \beta]$ $(j=1,2, \ldots, k)$ such that

$$
[\alpha, \beta] \subset \bigcup_{j=1}^{k} U\left(x_{j}, \delta\left(x_{j}\right)\right)
$$

Put $\delta=\min \left\{\delta\left(x_{j}\right) \mid j=1,2, \ldots, k\right\}$. Clearly, $\delta>0$. Take $x, y \in[\alpha, \beta]$ such that $(y-x) / h \in \mathbb{Q}^{+}$and $|y-x|<\delta$. Due to (5), there exists $m \in\{1,2, \ldots, k\}$ such that $x \in U\left(x_{m}, \delta\left(x_{m}\right)\right)$. Then

$$
\left.\frac{y-x}{h} \in \mathbb{Q} \cap\right] 0, \frac{\delta\left(x_{m}\right)}{|h|}[
$$

and $y=x+\frac{y-x}{h} h$. Hence, applying (4), we obtain

$$
\left|\frac{f(y)-f(x)}{(y-x) / h}-D_{h}^{\mathbb{Q}} f\left(x_{m}\right)\right|<\varepsilon .
$$

Thus we have

$$
|f(y)-f(x)|<\left(\left|D_{h}^{\mathbb{Q}} f\left(x_{m}\right)\right|+\varepsilon\right) \frac{|y-x|}{|h|} \leq(M+\varepsilon) \frac{|y-x|}{|h|} .
$$

Let us consider $u, v \in[\alpha, \beta]$ that satisfy $(v-u) / h \in \mathbb{Q}$. We may assume, without loss of generality, that $(v-u) / h>0$. Take $n \in \mathbb{N}$ with $n \delta>|v-u|$ and define $y_{j}=u+(j / n)(v-u)(j=0,1, \ldots, n)$. Then $\left|y_{j}-y_{j-1}\right|=|v-u| / n<\delta$ and $\left(y_{j}-y_{j-1}\right) / h=(v-u) /(n h) \in \mathbb{Q}^{+}$for $j=1,2, \ldots, n$; so we can apply 
(6) to obtain

$$
\begin{aligned}
|f(v)-f(u)| & =\left|\sum_{j=1}^{n}\left(f\left(y_{j}\right)-f\left(y_{j-1}\right)\right)\right| \leq \sum_{j=1}^{n}\left|f\left(y_{j}\right)-f\left(y_{j-1}\right)\right| \\
& \leq \sum_{j=1}^{n}(M+\varepsilon) \frac{y_{j}-y_{j-1}}{h}=\frac{M+\varepsilon}{h} \sum_{j=1}^{n}\left(y_{j}-y_{j-1}\right) \\
& =\frac{M+\varepsilon}{h}(v-u)=\frac{M+\varepsilon}{|h|}|v-u| .
\end{aligned}
$$

Letting $\varepsilon$ tend to 0 , we obtain (3).

Corollary 2.4. Suppose that $f: \mathbb{R} \rightarrow \mathbb{R}$ is strongly $\mathbb{Q}$-differentiable and $h \in \mathbb{R} \backslash\{0\}$ such that $D_{h}^{\mathbb{Q}} f(x)=0$ for every $x \in \mathbb{R}$. If $u, v \in \mathbb{R}$ satisfy $(v-u) / h \in \mathbb{Q}$, then $f(v)=f(u)$.

Proof. Take $u, v \in \mathbb{R}$ with $(v-u) / h \in \mathbb{Q}$ and choose $\alpha, \beta \in \mathbb{R}$ such that

$$
\alpha \leq \min \{u, v\} \text { and } \beta \geq \max \{u, v\} .
$$

Now we can apply Theorem 2.3 with $M=0$.

\section{Decomposition}

Strongly $\mathbb{Q}$-differentiable functions are characterized in the following theorem.

Theorem 3.1. A function $f: \mathbb{R} \rightarrow \mathbb{R}$ is strongly $\mathbb{Q}$-differentiable if, and only if, there exist a continuously differentiable function $g: \mathbb{R} \rightarrow \mathbb{R}$ and an additive mapping $\phi: \mathbb{R} \rightarrow \mathbb{R}$ such that

$$
f(x)=g(x)+\phi(x) \text { for every } x \in \mathbb{R} .
$$

Proof. Clearly, we have to prove the "only if" part. For this purpose, let us assume that $f: \mathbb{R} \rightarrow \mathbb{R}$ is strongly $\mathbb{Q}$-differentiable. Since the mapping $x \mapsto D_{1}^{\mathbb{Q}} f(x)$ is continuous by Proposition 2.2 , for $x \in \mathbb{R}$ we may define

$$
g(x)=f(0)+\int_{0}^{x} D_{1}^{\mathbb{Q}} f(s) d s .
$$

Then $g: \mathbb{R} \rightarrow \mathbb{R}$ is continuously differentiable and $g^{\prime}(x)=D_{1}^{\mathbb{Q}} f(x)$ for all $x \in \mathbb{R}$. 
Due to the examples and the note on the linear structure of strongly $\mathbb{Q}$-differentiable functions, the function $\phi=f-g$ is also strongly $\mathbb{Q}$-differentiable and

$$
D_{h}^{\mathbb{Q}} \phi(x)=D_{h}^{\mathbb{Q}} f(x)-D_{h}^{\mathbb{Q}} g(x)=D_{h}^{\mathbb{Q}} f(x)-g^{\prime}(x) h=D_{h}^{\mathbb{Q}} f(x)-D_{1}^{\mathbb{Q}} f(x) h
$$

for all $x, h \in \mathbb{R}$. In particular, $D_{1}^{\mathbb{Q}} \phi(x)=0$ for every $x \in \mathbb{R}$. Hence, by Corollary 2.4,

$$
\phi(x+s)=\phi(x) \text { for all } x \in \mathbb{R} \text { and } s \in \mathbb{Q} .
$$

Observing $\phi(0)=f(0)-g(0)=0$ and applying (7) with $x=0$, we obtain that $f(s)-g(s)=\phi(s)=0$ for every $s \in \mathbb{Q}$.

Our next statement is that the mapping $x \mapsto D_{h}^{\mathbb{Q}} \phi(x)$ is constant for every $h \in \mathbb{R}$. For this purpose, let us fix $h, x, y \in \mathbb{R}$ and consider sequences $\left(x_{n}\right)$ : $\mathbb{N} \rightarrow \mathbb{R},\left(r_{n}\right): \mathbb{N} \rightarrow \mathbb{Q}^{+}$such that $x=\lim _{n \rightarrow \infty} x_{n}$ and $0=\lim _{n \rightarrow \infty} r_{n}$. For every $n \in \mathbb{N}$, we can choose $s_{n} \in \mathbb{Q} \cap U\left(y-x_{n}, 1 / n\right)$. Thus $y=\lim _{n \rightarrow \infty}\left(x_{n}+s_{n}\right)$ and, applying (7), we obtain

$$
\begin{aligned}
D_{h}^{\mathbb{Q}} \phi(x) & =\lim _{n \rightarrow \infty} \frac{\phi\left(x_{n}+r_{n} h\right)-\phi\left(x_{n}\right)}{r_{n}} \\
& =\lim _{n \rightarrow \infty} \frac{\phi\left(x_{n}+s_{n}+r_{n} h\right)-\phi\left(x_{n}+s_{n}\right)}{r_{n}}=D_{h}^{\mathbb{Q}} \phi(y) .
\end{aligned}
$$

For each $y \in \mathbb{R}$, define

$$
\psi_{y}(x)=\phi(x+y)-\phi(x) \quad(x \in \mathbb{R}) .
$$

Then $\psi_{y}$ is strongly $\mathbb{Q}$-differentiable and

$$
D_{h}^{\mathbb{Q}} \psi_{y}(x)=D_{h}^{\mathbb{Q}} \phi(x+y)-D_{h}^{\mathbb{Q}} \phi(x)=0 \text { for every } x, h \in \mathbb{R} .
$$

Then it follows from Corollary 2.4 that $\psi_{y}$ is a constant function. Hence there exists $\chi: \mathbb{R} \rightarrow \mathbb{R}$ such that

$$
\phi(x+y)-\phi(x)=\chi(y) \text { for every } x, y \in \mathbb{R} .
$$

Substituting $x=0$ into equation (8) we obtain that $\chi(y)=\phi(y)-\phi(0)=\phi(y)$ for every $y \in \mathbb{R}$; i.e., $\chi=\phi$. Thus equation (8) yields that $\phi$ is additive.

With the aid of this decomposition theorem one can transport various results concerning additive functions to strongly $\mathbb{Q}$-differentiable functions. We formulate only one typical application. 
Corollary 3.2. If $f: \mathbb{R} \rightarrow \mathbb{R}$ is strongly $\mathbb{Q}$-differentiable and $f$ is bounded from above (or below) on a measurable set of positive Lebesgue measure, then $f$ is continuously differentiable.

Proof. Since $\mathbb{R}$ is $\sigma$-compact, we conclude from our hypotheses that there exists a bounded (i.e., precompact) set $T$ of positive Lebesgue measure such that $f$ is bounded from above (or below) on $T$. By Theorem 3.1, there exist a continuously differentiable function $g: \mathbb{R} \rightarrow \mathbb{R}$ and an additive mapping $\phi: \mathbb{R} \rightarrow \mathbb{R}$ such that $f=g+\phi$ (with pointwise addition). Since $g$ is continuous, it is bounded on (the closure of) $T$. Therefore, $\phi$ is also bounded from above (or below) on $T$. As is well known [4, Theorem 9.3.1, p. 210], this property implies that $\phi(x)=c x$ for $x \in \mathbb{R}$ for some $c \in \mathbb{R}$ and thus $f=g+\phi$ is also continuously differentiable.

\section{Restricted Domain}

It is worth noting that our results can be reformulated and proved analogously for functions defined on an arbitrary real open interval. However, such a reformulation makes the description of our ideas a little bit more complicated, including additional constraints for most of the equations and inequalities. Therefore, it is reasonable to present our concepts and arguments for the simplest case and let the proper reformulation of them to those readers who are interested in the case of a restricted domain. In the rest of the paper we provide the definition and a few hints for that case.

Let $I \subset \mathbb{R}$ be a non-void open interval. For every $h \in \mathbb{R}$ define

$$
T(I, h)=\left\{(x, r) \in I \times \mathbb{Q}^{+} \mid x+r h \in I\right\} .
$$

Let us note that, for every $h \in \mathbb{R}, T(I, h)$ is an open subset of $\mathbb{R} \times \mathbb{Q}^{+}$with respect to the topology induced by the restriction of the Euclidean topology to $\mathbb{R} \times \mathbb{Q}^{+}$.

We say that $f: I \rightarrow \mathbb{R}$ is strongly $\mathbb{Q}$-differentiable if the finite limit

$$
D_{h}^{\mathbb{Q}} f\left(x_{0}\right)=\lim _{T(I, h) \ni(x, r) \rightarrow\left(x_{0}, 0\right)} \frac{f(x+r h)-f(x)}{r}
$$

exists for every $x_{0} \in I, h \in \mathbb{R}$.

The reformulation of Example 1.1 for this case is quite simple. One has to be a little bit more careful with Example 1.2. Namely, if $A: \mathbb{R} \rightarrow \mathbb{R}$ is additive and $\phi: I \rightarrow \mathbb{R}$ is defined by $\phi(x)=A(x)$ for $x \in I$, then $\phi$ is strongly $\mathbb{Q}$-differentiable and $D_{h}^{\mathbb{Q}} \phi\left(x_{0}\right)=A(h)$ for every $x_{0} \in I, h \in \mathbb{R}$. 
Now it is easy to reformulate Theorem 3.1: a function $f: I \rightarrow \mathbb{R}$ is strongly $\mathbb{Q}$-differentiable if, and only if, there exist a continuously differentiable function $g: I \rightarrow \mathbb{R}$ and an additive mapping $A: \mathbb{R} \rightarrow \mathbb{R}$ such that $f(x)=g(x)+A(x)$ for every $x \in I$. The proof of this theorem involves the same argument as that of Theorem 3.1, including auxiliary results on the analogy of Propositions 2.1, 2.2 and Theorem 2.3, with a little difference if $0 \notin I$. Namely, we may define $g: I \rightarrow \mathbb{R}$ by

$$
g(x)=f\left(r_{0}\right)+\int_{r_{0}}^{x} D_{1}^{\mathbb{Q}} f(s) d s \quad(x \in I),
$$

where $r_{0} \in \mathbb{Q} \cap I$ is fixed. Then, following the same argument, we obtain that $\phi=f-g$ fulfills $\phi(u)=0$ for every $u \in \mathbb{Q} \cap I$ and there exists $\chi: I-I \rightarrow \mathbb{R}$ satisfying

$$
\phi(x+y)-\phi(x)=\chi(y) \text { for every } x \in I, y \in I-x
$$

(where $I-x=\{z-x \mid z \in I\}$ and $I-I=\{z-x \mid x, z \in I\}$ ). Applying an extension theorem [1, Theorem 1] concerning Pexider's equation, we obtain that there exist an additive mapping $A: \mathbb{R} \rightarrow \mathbb{R}$ and a real number $b \in \mathbb{R}$ such that $\phi(x)=A(x)+b$ for $x \in I$ and $\chi(y)=A(y)$ for $y \in I-I$. Using the property that $\phi$ vanishes at the rationals in $I$, we can easily show that $b=0$.

The statement and the proof of Corollary 3.2 also remain valid for functions defined on $I$.

Acknowledgement. The author is grateful to the referee for a valuable suggestion concerning the proof of the main theorem.

\section{References}

[1] J. A. Baker and F. Radó, Pexider's equation and aggregation of allocations, Aequationes Math. 32 (1987), 227-239.

[2] N. G. de Bruijn, Functions whose differences belong to a given class, Nieuw Arch. Wiskunde (2) 23 (1951), 194-218.

[3] A. Gilányi, A characterization of monomial functions, Aequationes Math. 54 (1997), 289-307.

[4] Marek Kuczma, An Introduction to the Theory of Functional Equations and Inequalities, Państwowe Wydawnictwo Naukowe, Warszawa-KrakówKatowice, 1985. 
[5] K. Nikodem and Zs. Páles, A characterization of midpoint-quasiaffine functions, Publ. Math. Debrecen 52/3-4 (1998), 575-595.

[6] A. Simon and P. Volkmann, Eine Charakterisierung von polynomialen Funktionen mittels der Dinghasschen Intervall-Derivierten, Results in Math. 26 (1994), 382-384. 